\title{
Physical Activity Behavior Change: Before, during and after the Lockdown (Covid 19) in Five Country
}

\author{
Lv Wan Gang, Zhou Xue Jun and Rahmat Hidayat*
}

Physical Education, Wuhan Sports University, China

*Corresponding author: Rahmat Hidayat, Physical Education, Wuhan Sports University, Wuhan, China

\begin{abstract}
Purpose: There are two purposes in this study (1) To identify whether self-determination factors can influence physical activity behavior in different situations, before, during, and after lockdown. (2) Whether self-determination factors can affect the frequency of physical activity, before, during and after lockdown.

Method: $B P A Q$ and BREQ-2 to 2.326 people China $\mathrm{n}=$ 607 Indonesia $n=1017$ Malaysia $n=341$ Philipina $n=127$ Vietnam $n=234$.

Result: BPAQ Intensity of Physical Activity, the highest percentage of LIT in during lockdown $54.37 \%$. MIT in after lockdown $56.57 \%$. HIT in before lockdown $24.99 \%$. Duration of Physical Activity, the highest percentage before lockdown 2-3 hours 33.16\%. During lockdown 1-2 hours 49.75\%. After lockdown 1-2 hours 45.99\%. Physical Activity in One Week, the highest percentage before lockdown 2-3 times $41.04 \%$. During lockdown 3-4 times 39.42\%. After lockdown 3-4 times 38.14\%. BREQ-2 Before lockdown Amotivation 0.25, External 1.49, Introjected 1.84, Identified 3.17, Intrinsic 3.50. During lockdown Amotivation 0.13, External 1.68, Introjected 2.37, Identified 3.34, Intrinsic 3.22. After lockdown Amotivation 0.19, External 1.65, Introjected 2.31, Identified 3.35, Intrinsic 3.36.

Conclusion: Self-determination factors have an effect on physical activity behavior and therefore the frequency of physical activity in several things, before, during, and after lockdown
\end{abstract}

\section{Keywords}

Covid 19, Lockdown, Physical activity, Self-determination theory

\section{Background}

Covid nineteen may be a virus that causes metastasis infections (WHO). This virus has unfolded terribly quickly in the majority of countries. many countries have enforced policies to impose lockdowns [1]. With the increasing unfolding of the coronavirus, most countries have enforced the "stay at home" rule as a result that athletes and sportsmen cannot exercise as was common [1]. Covid nineteen is incredibly simple to pass from person to person and simply spreads to contact with AN infected person [2]. Covid nineteen is quickly propelling the pattern of human life, besides the approach to a lifetime of exercise. people unit asked to refrain from outside activities and self-isolate reception, and follow social distancing up-to-date with others (WHO). As long because the internment and stay-at-home rules are enforced, many folks cannot do the same old exercises, this could cause a decrease in physical skills. Physical exercise has a significant effect on physical and mental health, as well as preventing health problems, strengthening the system, and reducing tension [3]. Covid nineteen will have an effect on youngsters, infants, the old, and other people with weak immune systems, one in every one of the ways that to extend immunity is exercise. With the implementation of internment rules and occupy range in many countries, there are several changes within the frequency of physical exertion. All sports competitions are suspended and arranged sporting activities are prohibited [4].

As long because the internment rule is enforced by the govt, we have a tendency to square measure obligated to isolate reception, isolation will have a big impact on the US physically and mentally [5]. Isolation leads to an increase in body fat and a loss of muscle mass, as well as a reduced immune system, a lack of mental

Citation: Gang LW, Jun ZX, Hidayat R (2021) Physical Activity Behavior Change: Before, during and after the Lockdown (Covid 19) in Five Country. Int J Sports Exerc Med 7:205. doi.org/10.23937/2469$5718 / 1510205$

Accepted: November 11, 2021; Published: November 13, 2021

Copyright: (c) 2021 Gang LW, et al. This is an open-access article distributed under the terms of the Creative Commons Attribution License, which permits unrestricted use, distribution, and reproduction in any medium, provided the original author and source are credited. 
sharpness and toughness, insomnia, and depression [6]. It's vital for the US to stay doing physical activity throughout isolation. even if we have a tendency to square measure solely reception, our bodies and minds still have to be compelled to exercise so as for our organs to perform properly, stop weight gain and maintain morale throughout these tough times. Exercise will facilitate keep our system robust, not liable to infection [7]. Covid nineteen will cause folks to own totally different perceptions looking at their characteristics and may modification their motivation for physical activity [8]. Out of doors, physical activity is taken into account an awfully vital activity that may result in somebody's physical health, additionally as psychological stability [9]. Regular participation in sports brings a physiological state [10]. Sport has taken a prominent place among the many issues that Covid-19 has posed. Living with a contagious disease means being constantly exposed to debates about the social position of sport and the ways through which it should be permitted or forbidden and for as long as possible [11].

There unit world organization agency/people that|folks that|those that|those $\mathrm{WHO}$ get pleasure from personal exercise that does not would like contact with others and UN agency observe social distancing others UN agency still participate in cluster exercises as before, and it's thought of useful to analysis whether or not or not there unit changes in fashion in things where COVID-19 continues to unfold [8]. People are advised to avoid outside activities and self-isolate reception, as well as to practice social distancing, which reduces interaction with others (WHO). Internment interventions may have a negative physiological and psychological effect, which can be mitigated by physical program reception [12]. Participation in any kind of relatively physical activity or sport can well be a crucial component of maintaining a healthy lifestyle, and it is without a doubt a significant public health message for those who live in geographically isolated areas." Furthermore, routine and moderate physical activity has been shown to improve the body's response to metastasis infections such as COVID-19 [13]. Globally, the COVID-19 pandemic is regarded as one of the most straightforward danger sections among pandemic warning stages. The state of emergency was declared in the majority of regions, except Asia, North America, and Europe, and body orders were given to immediately shut down all facilities except those that were absolutely necessary, due to a sharp rise in the number of reported cases of infection and death., like hospitals and grocery stores, and national infrastructure that offers electricity or water (BBC).

In several countries in Asia, there is the implementation of a lockdown/stay at home/social distancing with different time periods, China started lockdown at the end of January 2020, Malaysia started lockdown in mid-March 2020, in the Philippines, the lockdown began in mid-March 2020, in Vietnam lockdown began in early April 2020. In Indonesia, largescale social restrictions were implemented in mid-April. Large-scale social restrictions area unit restrictions on sure activities applied by residents in a district. With the imposition of large-scale social restrictions, the government is obliged to close schools and workplaces, limit religious activities, to restrict activities in public places such as fitness centers, joint sports. However, some cities in a country continue to carry out their usual activities, only applying social distancing and are not allowed to gather more than two people (CNN) From the difference in the application of regulations in a country, of course, the daily activities that are carried out will definitely be different from the usual day, this will also have an impact on the frequency of physical activities carried out as usual. The difference in the duration of implementing lockdown/stay at home/social distancing in several countries will certainly be an interesting matter when discussing the implementation of a lockdown/ stay at home/social distancing. In China, the application of lockdown takes longer than in other countries, in Indonesia only applies large-scale social restrictions and limits activities in public places. The frequency of physical activity in each country compared to before the implementation of the lockdown regulation will be very different in each country.

Regular Physical Activity (PA) has been shown to be highly beneficial to one's wellbeing and to reduce the risk of a variety of negative health problems, such as coronary heart disease and type two polygenic disorder, and breast and carcinoma [14]. Because of these helpful effects, international PA pointers state that for increased health, adults ought to accumulate thirty min or additional of moderate-intensity PA for a minimum of five days per week [15]. Behavior is broadly outlined as something associate degree organism or living being will, which has actions, words, and manifestations of emotions and thoughts [16]. A bodily movement made by skeletal muscles that uses more energy than when a person is at rest is commonly known as physical activity action [17].

There are some models that are used more often than others; the four most well-known theories used in a PA sense are as follows. The Transtheoretical Model is based on the Social Psychological Features Theory (SCT), the principle of Planned Behavior (TPB), and the SelfDetermination Theory (SDT) (TTM). Without a doubt, the use of these ideas has significantly increased. our knowledge of the psychological factors and processes that affect $P A$ actions.

This study uses Self-Determination Theory (SDT) as a result of victimization this theory we are going to conclude the motives for doing PA. SDT is also a smart theory that was developed to clarify emotional, cognitive, and activity responses in the associate 
action domain [18]. The concept of self-determination, constant as a result of the transtheoretical model, will facilitate in understanding why individuals are unit concerned in physical sports activities and whether or not or not or not they're going to try this within the long term. the foremost usually proverbial motivations for individuals to possess interaction Physical activity has many goals: to improve or maintain health, to improve physical appearance, to have fun, to compete, to have social experiences, and to obtain psychological benefits. Motives that are influenced by an external psychological factor. People who unite significantly (ex. enjoyment, social, and psychological) can't be as powerful as those who unite feature regulators (ex. improving physical appearance) [19].

During the implementation of lockdown/stay at home, physical activity behavior is likely to change from, as usual, limitations in using public facilities such as jogging tracks, fitness centers, sports halls, etc. Become an important point that can change PA behavior, besides that during the implementation of a lockdown/stay at home Everyone is advised to maintain their immunity so they are not susceptible to COVID 19, therefore it is advisable to keep the body's immunity to continue doing PA during lockdown/stay at home. Motivation in doing PA will of course be different from each stage before, during, and after the implementation of lockdown/stay at home. Before, during, and after implementing lockdown/stay-athome, the frequency of conducting PA will also be different. The aim of this study, (a) What self-determination factors influence changes in physical activity behavior in different situations, before, during, and after lockdown/stay at home (covid19)? (b) Can self-determination factors affect the frequency of physical activity before, during, and after lockdown/stay at home (covid 19)?

\section{Research Design}

This analysis was conducted pattern survey ways and questionnaires as a tool for conducting surveys. This research is a broad cross-sectional online survey that was completed using the Google web survey tool (google LLC, mountain browse, CA, United States) We collect data from 10th November to 10th December 2020. This online survey is distributed via, whats app, Instagram, Facebook, and WeChat. Before filling out the shape, the participants explained that each data is confidential and its confidentiality is secured. All participants gave their consent before collaborating [3].

\section{Participants}

Participants in this study were randomized in five countries. China 607 respondents 418 male and 189 female. Indonesia 1017 respondents 712 male and 305 female. Malaysia 341 respondents 205 male and 136 female. Philipina 127 participants 52 male and 75 female. Vietnam 234 participants 148 male and 86 female.

\section{Instruments}

Baecke Physical Activity Questionnaire (BPAQ) [20]. The Baecke questionnaire asks questions regarding sports activities, all based on frequency (how many times) per week, How many hours per day. For questions about sport and exercise, perceived intensity is included (as low, moderate, or high).

Behavioral control Behaviour Regulation Exercise kind (BREQ-2) provides the inspiration for physical activity. It was created by Mulland, Markland, and Ingledew [21] and consists of eighteen items. It is of the Likert kind, and it is divided into many sub-classes: Motivation (I don't see why I should exercise), external regulation (I don't see why I should exercise), and internal regulation (I don't see why I should exercise) (ex. I exercise because people tell me I should), integrated regulation (ex. I feel bad if I don't exercise), illustrious regulation (ex. I exercise because it's good for my health and looks good), and intrinsic motivation (ex. I exercise because it's fun).

\section{Statistical Analysis}

The basic descriptive statistical parameters, arithmetic mean and standard deviation, were calculated using IBM SPSS ${ }^{\circ}$ version 19 software and analysis of variance one-way ANOVA. The KolmogorovSmirnov test is used to determine if the variables have a normal distribution.

\section{The Intensity of Physical Activity}

Before the implementation of the lockdown in China, low-intensity exercise was $1.32 \%$, moderate-intensity exercise was $20.76 \%$ and high-intensity exercise was $77.92 \%$. During the low-intensity exercise lockdown, there was an increase of $2.47 \%$ and a decrease of $0.17 \%$ after the lockdown. Moderate intensity training during lockdown saw an increase of $75.45 \%$ and an increase of $29.66 \%$ after lockdown. There was a $29.49 \%$ decrease in high-intensity training after lockdown but there was a drastic decrease in high-intensity training during lockdown $0 \%$.

Low-intensity training before the implementation of PSBB in Indonesia was $10.42 \%$, moderate-intensity exercise was $53.18 \%$ and high-intensity exercise was $36.40 \%$. Low-intensity training during the implementation of the PSBB increased by $55.76 \%$ and $3.84 \%$ after the implementation of the PSBB. There was a decrease of $19.36 \%$ for moderate-intensity training during the implementation of the PSBB and an increase of $9.85 \%$ after the implementation of the PSBB. There was a decrease in high-intensity training by $13.69 \%$ after the implementation of the PSBB and a drastic decrease of $0 \%$ during the implementation of the PSBB.

In Malaysia, low-intensity exercise before lockdown is $45.16 \%$, moderate-intensity exercise is $50.15 \%$ and high-intensity exercise is $4.69 \%$. Low-intensity training 
during the implementation of the locking was an increase of $38.12 \%$ compared to before the implementation of the locking, however, after the implementation of the locking, there was a decrease of $20.23 \%$ when compared to the time of implementing the locking. Moderate intensity training decreased by $33.43 \%$ during the implementation of the lockdown and $13.20 \%$ after the implementation of the lockdown when compared to before the implementation of the lockdown. Highintensity training before implementation of the lockdown was $4.69 \%$ and decreased during and after the implementation of the lockdown $0 \%$.

Prior to the implementation of the lockdown, low-intensity exercise in the Philippines was 66.14\%, moderate-intensity exercise was $33.86 \%$ and highintensity exercise was $0 \%$. After the implementation of the locking exercise with low intensity there was a decrease of $22.05 \%$ and an increase of $15.75 \%$ during the implementation of the locking exercise. Moderate intensity training after the implementation of the lockdown increased by $22.05 \%$ and decreased by $15.75 \%$ during the implementation of the lockdown.

In Vietnam, low-intensity exercise before implementation of lockdown is $14.53 \%$, moderateintensity exercise is $79.49 \%$ and high-intensity exercise is $5.98 \%$. Low-intensity training experienced a decrease of $2.99 \%$ after implementing the lockdown and an increase of $22.22 \%$ during the implementation of the lockdown when compared to before the implementation of the lockdown. There was a decrease in moderate-intensity training by $16.24 \%$ during the implementation of the lockdown and $2.99 \%$ after the implementation of the lockdown. High-intensity training was the same as in other countries when the lockdown was implemented in Vietnam, namely $0 \%$, but there was an increase of $5.98 \%$ after the implementation of the lockdown.

From the data of 5 countries during the implementation of the lockdown for low-intensity training, all of them increased, only one country for moderate-intensity training had increased, while all high-intensity exercises experienced a decrease. After the implementation of lockdown for low-intensity exercise in two countries increased, moderate-intensity exercise for 3 countries increased and high-intensity exercise for only one country increased.

\section{Duration of Physical Activity in a Day}

In China, the duration of exercise before lockdown is $1-2$ hours $1.81 \%, 2-3$ hours $38.22 \%, 2-4$ hours $35.75 \%$ and $>4$ hours $24.22 \%$. during the implementation of lockdown 1-2 hours $28.66 \%$, 2-3 hours $60.63 \%$, 3-4 hours $9.39 \%$, > 4 hours $1.32 \%$. after applying lockdown 1-2 hours $2.80 \%, 2-3$ hours $20.76 \%$, 3-4 hours $61.12 \%$ and $>4$ hours $15.32 \%$.

In Indonesia, the duration of training before the implementation of lockdown $<1$ hour 7.97\%, 1-2 hours
$29.20 \%, 2-3$ hours $42.38 \%$, 3-4 hours $17.30 \%$, > 4 hours $3.15 \%$. During the implementation of lockdown $<1$ hour $35.10 \%, 1-2$ hours $47.30 \%, 2-3$ hours $9.04 \%$, 3-4 hours $6.88 \%$ and $>4$ hours $1.68 \%$. After applying lockdown < 1 hour $3.04 \%$, 1-2 hours $56.62 \%$, 2-3 hours $33.51 \%$, 3-4 hours $4.50 \%$, > 4 hours $2.33 \%$.

In Malaysia, the duration of training before the implementation of lockdown $<1$ hour is $12.02 \%, 1-2$ hours $72.43 \%$, $2-3$ hours $15.55 \%$. As long as the lockdown is implemented $<1$ hour $7.62 \%, 1-2$ hours $82.40 \%, 2-3$ hours $9.98 \%$. after applying lockdown $<1$ hour $14.96 \%$, $69.20 \% 1-2$ hours and $15.84 \%$ 2-3 hours.

In the Philippines, the duration of training before the implementation of lockdown $<1$ hour was $63.78 \%, 1-2$ hours $36.22 \%$. as long as the lockdown is implemented $<1$ hour 22.83\%, 1-2 hours 77.17\%. After applying lockdown $<1$ hour $15.75 \%, 1-2$ hours $84.25 \%$.

In Vietnam, the training duration before implementing the lockdown is $1-2$ hours $24.79 \%$, 2-3 hours $69.66 \%, 3-4$ hours $5.55 \%$. During the implementation of lockdown 1-2 hours $13.24 \%, 2-3$ hours $79.50 \%$, and 3-4 hours $7.26 \%$. After applying lockdown 1-2 hours $17.09 \%, 2-3$ hours $73.94 \%$, and 3-4 hours $8.97 \%$.

\section{Physical Activity in One Week}

The number of exercises in one week in China before the implementation of lockdown 2-3 times a week $19.93 \%, 3-4$ times a week $62.44 \%$, 4-5 times a week $11.54 \%$, > 5 times a week $6.09 \%$. During the implementation of lockdown 2-3 times a week decreased to $2.14 \%, 3-4$ times a week it decreased to $14.33 \%$, 4-5 times a week increased to $71.00 \%$, and $>5$ times a week increased to $12.53 \%$. after applying lockdown 2-3 times a week 2.47\%, 3-4 times $8.90 \%$ a week, 4-5 times a week $79.24 \%$ and $>5$ times a week $9.39 \%$. Before the implementation of the lockdown, people in China did a lot of physical activity 3-4 times a week. During the implementation of the lockdown, the community did more physical activity 4-5 times a week. Even after the lockdown was carried out, people who did physical activity 4-5 times a week increased to $79.24 \%$.

In Indonesia, before the implementation of lockdown once a week it was 7.87\%, 1-2 times a week $26.64 \%, 2-3$ times a week $38.94 \%$, 3-4 times a week $13.38 \%$, a week 4-5 times $13,17 \%$ and $>5$ times a week $6.09 \%$. During the implementation of lockdown 1-2 times a week 11.20\%, 2-3 times a week $23.30 \%$, 3-4 times a week $27.15 \%$, a week $4-5$ times $31.57 \%$, and $>5$ times a week 6.78. after applying lockdown once a week 5.11\%, a week 1-2 times $15.34 \%$, 2-3 times a week $17.30 \%$, a week 3-4 times $41.89 \%$, a week 4-5 times $20.36 \%$. Before implementing the lockdown, $38.94 \%$ of Indonesians did physical activity 2-3 times a week. During the implementation of the lockdown, Indonesians did more physical activity 4-5 times a week. After the lockdown was implemented, $41.89 \%$ of the people did physical activity 3-4 times a week. 
In Malaysia before the implementation of lockdown 1-2 times a week $14.37 \%, 2-3$ times a week $79.48 \%, 3-4$ times a week $6.15 \%$. During the implementation of the lockdown 2-3 times a week 16.71\%, 3-4 times a week 83.29\%. After applying lockdown 2-3 times a week 48.09\%, 3-4 times a week 51.91\%. Before the lockdown was implemented $79.48 \%$ of Malaysians did physical activity 2-3 times a week. During the implementation of the lockdown, they did physical activity more than 3-4 times a week, namely $83.29 \%$. After the lockdown, most people did a physical activity which remained the same, namely 3-4 times a week but decreased to $51.91 \%$.

In the Philippines before the implementation of lockdown 1-2 times a week $57.48 \%$, 2-3 times a week $42.52 \%$. provided that the lockdown is implemented $1-2$ times a week $29.92 \%$, 2-3 times a week $70.08 \%$. after applying lockdown 1-2 times a week $18.90 \%$, 2-3 times a week $81.10 \%$. Before implementing the lockdown, most people did physical activity 1-2 times a week. During the implementation of the lockout 2-3 times a week. After the lockdown, most people did physical activity regularly, 2-3 times a week, but this increased to $81.10 \%$.

In Vietnam, before the implementation of lockdown 2-3 times, a week was $24.36 \%$, 3-4 times a week $62.39 \%$, 4-5 times a week $13.25 \%$. during the implementation of lockdown 2-3 times a week $9.40 \%, 3-4$ times a week $72.23 \%$, 4-5 times a week $18.37 \%$. After applying lockdown 2-3 times a week 5.98\%, 3-4 times a week $88.04 \%$, a week $4-5$ times $5.98 \%$. Most of the physical activities were carried out by the community before, during, and after the implementation of the lockdown, namely 3-4 times a week but there was an increase of $9.48 \%$ during the implementation of the lockdown and $25.65 \%$ after the implementation of the lockdown when compared before implementing the lockdown.

\section{Result of BREQ-2}

From the data obtained before the implementation of the lockdown in China, the motivation factor got the lowest score and the highest score was the intrinsic motivation factor, but when the implementation of the Introjected and Identified lockdown increased the lowest value remained motivation but the highest value was the identified factor. After the implementation of the lockdown, the introjected factor increased compared to before and through the implementation of the imprisonment. the best issue once the implementation of the lockdown was intrinsic but decreased by 0.04 when compared to before the implementation of the lockdown. One way ANOVA motivation test results before and during lockdown implementation $P=0.04$, during and after lockdown application $P=0.01$, before and after lockdown application $P=0.00$. From the data obtained, there are significant differences between the three. External regulations before and during lockdown implementation $\mathrm{P}=0.46$, during and after lockdown implementation $P=0.66$, before and after lockdown implementation $P=0.20$. From the data obtained, there is no significant difference between the three. Introduced before and during locking $P=0.00$, during and after locking $P=0.00$, before and after locking $P$ $=0.00$. From the data obtained, there are significant differences between the three. The regulations identified before and during the implementation of the lockdown $P=0.00$, during and after the implementation of the lockdown $P=0.00$, before and after the implementation of the lockdown $P=0.00$. From the data obtained, there are significant differences between the three. Intrinsic regulation before and during lockdown implementation $P=0.00$, during and after lockdown implementation $P$ $=0.00$, before and after lockdown implementation $P$ $=0.00$. From the data obtained, there are significant differences between the three.

Before the implementation of the PSBB, the intrinsic factor was the highest value and the motivation factor was the lowest value, as long as the implementation of the PSBB, external, introjection, identified, and intrinsic factors increased but the most introjected factor increased, namely 0.83 . after the implementation of PSBB the Identified Factors increased by 0.10 . One way ANOVA motivation test results before and during lockdown implementation $P=0.00$, during and after lockdown application $P=0.00$, before and after lockdown application $P=0.00$. From the data obtained, there are significant differences between the three. External regulations before and during lockdown implementation $\mathrm{P}=0.00$, during and after lockdown implementation $\mathrm{P}=$ 0.37 , before and after lockdown implementation $P=0.00$. From the data obtained, there are significant differences before and during the implementation of lockdown andbefore and after the lockdown was implemented, but during and There is no discernible difference after the introduction of lockdown. Introduced before and during locking $P=0.00$, during and after locking $P=$ 0.00 , before and after locking $P=0.00$. From the data obtained, there are significant differences between the three. Identification of regulations before and during the implementation of the lockdown $P=0.00$, during and after the implementation of the lockdown $P=0.13$, before and after the implementation of the lockdown $P$ $=0.00$ from the data obtained that there were incidents both before and after the lockout, and that there were incidents both before and after the lockdown. There is a big difference. difference, however, during and after the implementation of the lockdown there is no significant difference. Intrinsic regulation before and during lockdown implementation $P=0.00$, during and after lockdown implementation $P=0.00$, before and after lockdown implementation $P=0.00$. From the data obtained, there are significant differences between the three.

Before the implementation of lockdown, the intrinsic factor was the highest factor, as long as external, introjection, and identified factors were locked, 
identified as the highest factor and after lockdown, the identified factors remained the highest and increased by 0.09 . One way ANOVA motivation test results before and during lockdown implementation $\mathrm{P}=0.00$, during and after lockdown application $P=0.00$, before and after lockdown application $P=0.00$. From the data obtained, there are significant differences between the three. External regulations before and during lockdown implementation $P=0.29$, during and after lockdown implementation $\mathrm{P}=0.47$, before and after lockdown implementation $P=0.74$. From the data obtained, there is no significant difference between the three. Introduced before and during locking $P=0.00$, during and after locking $P=0.00$, before and after locking $P$ $=0.00$. From the data obtained, there are significant differences between the three. The regulations identified before and during the implementation of the lockdown $P=0.00$, during and after the implementation of the lockdown $\mathrm{P}=0.00$, before and after the implementation of the lockdown $P=0.00$ from the data, there was a significant difference. between the three. Intrinsic regulation before and during lockdown implementation $\mathrm{P}=0.00$, during and after lockdown implementation $\mathrm{P}$ $=0.00$, before and after lockdown implementation $P$ $=0.00$. From the data obtained, there are significant differences between the three.

Before implementing the lockdown, the Philippines was the highest factor. During the implementation of the lockdown, external factors were introduced and identified but external factors increased by 0.16 to the maximum. After the implementation of lockdown, the intrinsic factor becomes the highest factor. One way ANOVA motivation test results before and during lockdown implementation $P=0.11$, during and after lockdown application $P=0.76$, before and after lockdown application $P=0.00$. From the data obtained, it is known that before and during the implementation of the lockdown as well as during and after the implementation of the lockdown there is no significant difference, but before and after the implementation of the lockdown there are significant differences. External regulation before and during lockdown implementation $P=0.02$, during and after lockdown implementation $P=0.58$, before and after lockdown implementation $P=0.00$. There are substantial variations in the data collected before and after the implementation of lockdown, as well as before and after the implementation of lockdown, but there are no significant differences during and after the implementation of lockdown. there is no significant difference. Introduced before and during locking $P=0.07$, during and after locking $P=0.77$, before and after locking $P=0.03$. From the data obtained, there is no significant difference before and during the implementation of lockdown as well as during and after the implementation of lockdown, but before and after the implementation of lockdown there are significant differences. The regulations identified before and during the implementation of the lockdown $P=0.45$, during and after the implementation of the lockdown $P=0.59$, before and after the implementation of the lockdown $P=0.83$ from the data it was found that there was no significant difference between the three. Intrinsic regulation before and during lockdown implementation $P=0.18$, during and after lockdown implementation $P$ $=0.09$, before and after lockdown implementation $\mathrm{P}$ $=0.74$. From the data obtained, there is no significant difference between the three.

Vietnam before implementing lockdown, the intrinsic factor is the highest factor. During the implementation of a lockdown, the factor was identified as the highest factor and after the implementation of the lockdown, the intrinsic factor was the highest factor. One way ANOVA motivation test results before and during lockdown implementation $\mathrm{P}=0.00$, during and after lockdown application $P=0.00$, before and after lockdown application $P=0.01$. From the data obtained, there are significant differences between the three. External regulations before and during lockdown implementation $P=0.00$, during and after lockdown implementation $P$ $=0.35$, before and after lockdown implementation $P$ $=0.00$. From the data obtained, there are significant differences before and during the implementation of lockdown and there is no significant difference between before and after the implementation of lockdown, but there is no significant difference between before and after the implementation of lockdown. Introduced before and during locking $P=0.00$, during and after locking $P=0.02$, before and after locking $P=0.00$. From the data obtained, there are significant differences between the three. Identification of regulations before and during lockdown implementation $P=0.00$, during and after lockdown implementation $P=0.14$, before and after lockdown implementation $P=0.00$ from the data obtained that before and during lockdown implementation and before and after lockdown implementation there was a significant difference but during and after the implementation of the lockdown there was no significant difference. Intrinsic regulation before and during lockdown implementation $P=0.00$, during and after lockdown implementation $P=0.00$, before and after lockdown implementation $P=0.02$. From the data obtained, there are significant differences between the three.

\section{Discussion}

There are two purposes of this research. The first is to determine whether self-determination factors can influence changes in physical activity in various situations, before, during, and after the lockdown. And second, whether self-determination factors affect the frequency of physical activity before, during, and after the lockdown. From the data obtained, self-determination factors can influence changes in physical activity before, during, and after the application of a lock. Before the 
lockdown was carried out, the motivation factor was the lowest factor and the highest factor was the intrinsic factor. The results of this study are the same as previous studies [22] that all motivation regulators are on one continuum in the low to high (intrinsic) motivation range. During the implementation of the lockdown, the motivation factor remains the lowest factor but the highest factor is identified in China and Malaysia. After the implementation of the lockdown, the motivation factor remains the lowest factor, but in Indonesia and Malaysia, the highest factor is identified.

In China, Prior to the introduction of lockdown, the intrinsic factor was the most important factor, followed by training frequency (the most). 2-3 hours of highintensity exercise a day, 3-4 days a week. During the implementation of the lockdown, the factors that were highest were identified with the frequency of training (at most) moderate-intensity training, 2-3 hours per day, and 4-5 times a week. After the implementation of lockdown, the intrinsic factor became the highest factor with the frequency of training (at most) moderateintensity training, 3-4 hours per day and 4-5 times a week. In Indonesia, before the implementation of the lockdown, the intrinsic factor was highest with the frequency of training (at most) moderate-intensity training, 2-3 hours per day and 2-3 times a week. During the implementation of the lockdown, the intrinsic factor was the highest factor with the lowest intensity training frequency,1-2 hours a day, 4-5 days a week Following the lockdown, the most important variables were described as the duration of moderate-intensity training (most), 1-2 hours a day, and 3-4 days a week.

In Malaysia, before the implementation of the lockdown, the intrinsic factor was the highest factor with the frequency of training (at most) moderateintensity training, 1-2 hours a day, 2-3 days a week is recommended. During the lockdown implementation, the factors identified with the highest training frequency (at most) were low-intensity exercise, 1-2 hours per day, and 3-4 times a week. After implementing the lockdown, the factors identified with the highest training frequency (at most) were low-intensity exercise, 1-2 hours a day, three or four days a week.

In the Philippines before, during, and after the implementation of the lockdown, the intrinsic factor was the highest. Prior to the implementation of the lockdown, the training frequency (at most) was lowintensity exercise, < 1 hour per day and 1-2 times a week. During the implementation of the lockdown, the training frequency (at most) was low-intensity exercise, 1-2 hours a day, 2-3 days a week is recommended. After the lockdown, the training will consist of (at most) 1-2 hours of moderate-intensity exercise, 2-3 days a week.

In Vietnam, before, during, and after the implementation of lockdown, the intrinsic factor was the highest factor with the (highest) training frequency for moderate-intensity training, 3-4 days a week and 2-3 hours a day. From each of the different situations before, during, and after the implementation of the lockdown, there are significant differences which can be seen.

\section{Conclusion}

The existence of different situations before, during, and after the implementation of lockdowns can change the self-determination factor so that it can affect the frequency of physical activity. Based on the findings of this study and the Theory of Self-Determination, the element of self-determination is a significant factor that can affect a person's decision to engage in physical activity. The results of this study and previous research can be used as a reference for physical activity. Covid-19 can change all aspects of life including physical activity behavior, therefore it is important to continue to do a physical activity so that the body remains healthy and has good endurance. In addition, when a person is more active in physical activity, he will get psychological benefits in the form of reducedstress, anxiety, and depression levels We also have to participate in sporting events during Covid 19. but still have to maintain health and comply with health regulations, use masks, wash our hands more often and maintain a minimum distance of 1 meter from other people.

\section{Perspective}

In this study, it was found that intrinsic and identifiable factors are the highest self-determination factors that can effect changes in a person's physical activity, in previous studies only intrinsic factors, are the highest factors that can change physical activity behavior [21]. In measuring the Amotivation, External, Introjected, Identified, and Intrinsic factors in China and Malaysia, there is one factor that does not change significantly namely external factors. In Joan B Landry's research, External factors, it was explained, had no major impact on a person's desire to engage in physical activity. In Indonesia and Vietnam, the external factors and factors identified during and after the implementation of the lockdown did not have a significant difference. In the Philippines, the motivational and external factors experienced significant alterations Just before and after the lockdown did the predicted variables experience significant changes, and only before and after the lockdown did they experience significant changes.

\section{Limitation}

There are limitations in this study, namely the sample size is not the same between countries, educational background or work is not the same. The same number of samples and background samples will provide a solid basis. In this study, physical status and health were not assessed and could be an important factor in physical activity. In addition, Anxiety factors can influence a person's ability to engage in physical activity when a lockdown is implemented. Further research is needed 
to look at physical status and fitness, as well as the level of anxiety that influences physical activity.

\section{References}

1. Gilat R, Cole BJ (2020) COVID-19, Medicine and Sports 2: 175-176.

2. McDermott D, Firth J, Grabovac I, Yakkundi A, Armstrong $\mathrm{N}$, et al. (2020) A cross-sectional analysis during the implementation of COVID-19 social distancing found a correlation between physical activity and psychological condition in a large sample of the UK public. Ment Health Phys Act 19: 100345.

3. Schnitzer M, Schobersberger W, Weiss G, Frühauf MK (2003) Inter-disciplinary guidelines to keep people physically healthy during the covid-19 internment in the state, Austria, include jogging, nordic cycling, and going for a stroll. Polski Merkuriusz Lekarski 14: 557-558.

4. Zheng YY, Ma YT, Zhang JY, Xie X (2020) Soccer cannot resume at this time due to the COVID-19 emergency!A critical viewpoint from Italian expertise and a concern action. Nat Rev Cardiol 17: 259-260.

5. Jukic J, Calleja-González F, Cos F, Cuzzolin J, Olmo N, et al. (2020) COVID-19 provides forms and solutions for team sports athletes who are alone. Sports 8: 56 .

6. Chen P, Mao L, Nassis GP, Harmer P, Ainsworth BE, et al. (2020) Coronavirus illness (COVID-19): The need to maintain regular physical activity while taking precautions. J Sport Health Sci 9: 103-104.

7. Elmagd MA (2020) Sports and physical activity are encouraged throughout the (COVID-19) pandemic. 82-84.

8. Choi C, Bum CH (2020) Changes in the type of sports activity as a result of COVID-19: Hypochondria and thus the intention to continue participating in sports. Int J Environ Res Public Health 17: 4871.

9. Copper SL (2020) Promoting Physical Activity for Mental Well-Being. ACSMs Health Fit J 24: 12-16.

10. Booth FW, Roberts CK, Laye MJ (2012) Chronic diseases can be caused in part by a lack of exercise. Compr Physiol 2: $1143-1211$
11. Rowe D (2020) Pandemic sport is being subjected to a social science procedure. J Soc Sci.

12. Parnell D, Widdop P, Bond A, Wilson R (2020) COVID-19, networks, and sports are all things that come to mind when thinking about COVID-19. Managing Sport and Leisure 1-7.

13. Hammami A, Harrabi B, Mohr M, Krustrup P (2020) Specific guidelines for home-based physical coaching in relation to physical activity and coronavirus disease 2019 (COVID-19). Managing Sport and Leisure 1-6.

14. Shiroma EJ, Lobelo F, Puska P, Blair SN, Katzmarzyk PT, et al. (2012) The impact of physical inactivity on major noncommunicable diseases around the world: a study of disease burden of disease and life expectancy. The Lancet 380: 219-229.

15. Garber CE, Blissme B, Deschenes MR, Franklin BA, Lamonte MJ, et al. (2011) The Amount and Quality of Exercise for Developing and Maintaining Motor Fitness in Apparently Healthy Adults: Guidelines for Exercise Prescription. Med Sci Sports Exerc 43: 1334-1359.

16. Morris EK, Morris EK, Morris E (1992) Behavior Analysis: Its Purpose, Progress, and Evolution. Behav Anal 15: 3-29.

17. Leavitt's MO (2008) Physical Activity and Health in 2008 (San Francisco).

18. Downs DS, Nigg OR, Hausenblas HA, Rauff EL (2013) Why do people change their physical activity habits?. ACSMs Health Fit J 1-38.

19. Aleksovska-Velickovska L, Gontarev S, Ruzdija K (2019) Students' incentive to engage in physical activity: A selfdetermination theory. Int J Hum Sport Exerc 2.

20. Baecke JA, Burema J, Frijters JER (1982) In medical sciences, a short term for the practice of habitual physical activity. Am J Clin Nutr 36: 936-942.

21. Mullan E, Markland D (1997) Adults' self-determination varies as they progress through the phases of exercise adjustment. Motivation and feeling 21: 349-362.

22. Landry JB, Solmon MA (2004) African yank women's self-determination as it progresses through the phases of change for exercise. J Sport Exerc Psychol 26: 457-469. 